\section{Low Dose Radiation Therapy and Convalescent Plasma: How a Hybrid Method May Maximize Benefits for COVID-19 Patients}

\author{
Abdollahi H. ${ }^{1 \oplus}$, Shiri I. ${ }^{2}$, Bevelacqua J. J. ${ }^{3}$, Jafarzadeh A.4, \\ Rahmim A. ${ }^{5,6}$, Zaidi H..$^{2,7,8,9^{\circ}}$, Mortazavi S. A. R. ${ }^{10}$, Mortazavi \\ S. M. J. ${ }^{11 * \odot}$
}

\begin{abstract}
Physicians and scientists around the world are aggressively attempting to develop effective treatment strategies. The treatment goal is to reduce the fatality rate in $15 \%$ to $20 \%$ of individuals infected with SARS-CoV-2 who develop severe inflammatory conditions that can lead to pneumonia, and acute respiratory distress syndrome. These conditions are major causes of death in these patients. Convalescent plasma (CP) collected from patients recovered from the novel corona virus disease (COVID-19) has been considered as an effective treatment method for COVID-19. Moreover, low-dose radiation therapy (LDRT) for COVID-19 pneumonia was historically used to treat pneumonia during the first half of the $20^{\text {th }}$ century. The concept of LDRT for COVID-19 pneumonia was first introduced in March 2020. Later scientists from Canada, Spain, United States, Germany and France also confirmed the potential efficacy of LDRT for treatment of COVID-19 pneumonia. The rationale behind introducing LDRT as an effective treatment method for pneumonia in COVID-19 patients is not only due to its anti-inflammatory effect, but also in optimization of the activity of the immune system. Moreover, LDRT, unlike other treatment methods such as antiviral drugs, does not have the key disadvantage of exerting a significant selective pressure on the SARS-CoV-2 virus and hence does not lead to evolution of the virus through mutations. Given these considerations, we believe that a hybrid treatment including both CP and LDRT can trigger synergistic responses that will help healthcare providers in mitigating today's COVID-19 pandemic.
\end{abstract}

Citation: Abdollahi H, Shiri I, Bevelacqua JJ, Jafarzadeh A, Rahmim A, Zaidi H, Mortazavi SAR, Mortazavi SMJ. Low Dose Radiation Therapy and Convalescent Plasma: How a Hybrid Method May Maximize Benefits for COVID-19 Patients. J Biomed Phys Eng. 2020;10(4):387-394. doi: $10.31661 /$ jbpe.v0i0.2006-1125.

Keywords

Low Dose Radiation; Radiotherapy; COVID-19; Convalescent Plasma; AntiInflammatory Responses; Immune System; Selective Pressure; Mutations

\section{Introduction}

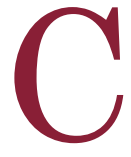

OVID-2019 disease is caused by a novel coronavirus known as SARS-CoV-2, and its pandemic was identified as an international public health crisis by the World Health Organization (WHO) $[1,2]$. SARS-CoV-2 has four main structural proteins; namely spike (S) glycoprotein, small envelope (E) glycoprotein, membrane (M) glycoprotein, and nucleocapsid $(\mathrm{N})$ protein, as well as several accessory proteins [1]. The $\mathrm{S}$ glycoprotein is surface-exposed and mediates entry into host cells through binding to its receptor called ACE2. The S protein
${ }^{1} \mathrm{PhD}$, Department of Radiolog ic Sciences and Medical Physics, Faculty of Allied Medicine, Kerman University of Medical

Science, Kerman, Iran

${ }^{2} \mathrm{PhD}$, Division of Nuclear

Medicine and Molecular

Imaging, Geneva University

Hospital, CH-1211 Geneva 4,

Switzerland

${ }^{3} \mathrm{PhD}$, Bevelacqua Resources,

Richland, Washington, United

States

${ }^{4} \mathrm{PhD}$, Department of Immu-

nology, School of Medicine,

Kerman University of Medical

Science, Kerman, Iran

${ }^{5} \mathrm{PhD}$, Departments of Radiol-

ogy and Physics, University of

British Columbia, Vancouver

$\mathrm{BC}$, Canada

${ }^{6} \mathrm{PhD}$, Department of Integra-

tive Oncology, BC Cancer

Research Centre, Vancouver

BC, Canada

${ }^{7} \mathrm{PhD}$, Geneva University Neu-

rocenter, Geneva University,

Geneva, Switzerland

${ }^{8} \mathrm{PhD}$, Department of Nuclear

Medicine and Molecular Imag

ing, University of Groningen,

University Medical Center

Groningen, Groningen, Neth-

erlands

${ }^{9} \mathrm{PhD}$, Department of $\mathrm{Nu}-$

clear Medicine, University of

Southern Denmark, Odense,

Denmark

${ }^{10} \mathrm{MD}$, School of Medicine,

Shiraz University of Medical

Sciences, Shiraz, Iran

${ }^{11} \mathrm{PhD}$, Department of Medical

Physics, School of Medicine,

Shiraz University of Medical

Sciences, Shiraz, Iran

*Corresponding author:

S. M. J. Mortazavi

Department of Medical

Physics, School of Medi-

cine, Shiraz University

of Medical Sciences,

Shiraz, Iran

E-mail: mortazavismj@ gmail.com

Received: 9 June 2020 Accepted: 19 June 2020 
is the main target for neutralizing antibodies upon infection and the focus of vaccine design [1]. The COVID-19-related clinical symptoms appear after an incubation phase of about 5-6 days. The time period from the beginning of the COVID-19 symptoms to death vary from 6-41 days, with a median of 14 days [3]. The clinical manifestations of SARS-CoV-2 infection have similarities with SARS-CoV as its most common symptoms include fever, dry cough, dyspnea, chest pain, fatigue, myalgia and bilateral pneumonia $[1,3,4]$. The severe pneumonia related to coronaviruses is usually characterized by rapid viral replication, extensive lung infiltration with inflammatory cells, elevated production of inflammatory mediators contributing to acute lung injury (ALI), and acute respiratory distress syndrome (ARDS), which may lead to death in some severe cases. Similar to MERS-CoV and SARS$\mathrm{CoV}$, there is still no specific antiviral treatment for COVID-19. Isolation and supportive care, including oxygen therapy, fluid management, and antibiotics treatment for secondary bacterial infections are recommended [5]. Although a wide range of therapeutic agents are being investigated, the mortality rates might increase [6]. As a new suggested treatment approach, convalescent plasma (CP) transfusion is receiving attention as a feasible way to treat patients. In addition, the US Food and Drug Administration (FDA) is supporting a national expanded treatment protocol to provide CP to COVID-19 patients across the country [7]. Besides CP, a wide array of research projects have been conducted on the impact of exposure to low doses of ionizing radiation (LDR) in the treatment of severe pneumonia in COVID-19 patients. While the suggested radiation doses range from $100 \mathrm{mGy}$ to $1 \mathrm{~Gy}$, anti-inflammatory effects of LDR and optimization of the immune system are the bases of these treatments. We believe that possible synergistic interactions of CP and LDR on the COVID-19 pathogenesis can justify an attempt to investigate the combinational effects of CP and LDR as a more effective treatment method.

Convalescent plasma can interfere with viral dissemination

It has been hypothesized that SARS-CoV-2 might pass through mucous membranes, especially nasal and larynx mucosa, with the virus subsequently entering the lungs through the respiratory tract. The virus may enter the peripheral blood from the lungs, causing blood viremia. Following this, the virus could attack target organs that express ACE2, including lungs, heart, kidneys, and gastrointestinal tract [8]. SARS-CoV-2 detected in fecal samples has revealed that the virus enters the blood from the lungs and then travels via blood to the intestines $[9,10]$. The expression and distribution of ACE2 in the human body may indicate potential infection routes of SARSCoV-2. High ACE2 expression was identified in type II alveolar cells (AT2) of lung, esophagus upper and stratified epithelial cells, absorptive enterocytes from ileum and colon, cholangiocytes, myocardial cells, kidney proximal tubule cells, and bladder urothelial cells [11]. COVID-19 also invades the central nervous system (CNS) to induce neurological abnormalities. SARS-CoV-2 may initially invade peripheral nerves and enter the CNS via the synaptic route [12]. Expression of ACE2 was reported in the CNS, which provides a route for SARS-CoV-2 entering into the brain [13]. A certain population of peripheral leukocytes are also infected by SARS-CoV-2 as they express ACE2 on their cell surface [14]. These findings indicate that ACE2-expressing tissues and cells should be considered as potential targets of SARS-CoV-2 infection [11]. Convalescent plasma originating from patients who have previously recovered from viral infection contain neutralizing antibodies against SARS-CoV-2. Once transfused into the patient, the antibodies from the CP are thought to neutralize the virus and limit its transmission. Accordingly, the protective antibody binds to the virus and prevents its attachment 
Radiation Therapy Plus Plasma for Treating COVID-19 Patients

to ACE2-expressing tissues and cells. The results from an exploratory meta-analysis of 32 studies showed evidence of reduced mortality after receiving various doses of $\mathrm{CP}$ in patients with severe acute respiratory infections of viral etiology [15]. The data collected by Chinese scientists show that human $\mathrm{CP}$ is a potential therapeutic option to decrease the severity and/or duration of the illness caused by COVID-19 [16].

CP collected from patients who have recovered from COVID-19 has been introduced as an effective treatment method against it. Given this consideration, in some countries, people who have fully recovered from COVID-19 for greater than 2 weeks are encouraged to donate their plasma to help saving lives. In three recent case studies, 19 COVID-19 patients were treated with CP from donors who had recovered from COVID-19 [17-19]. The results of these studies showed that CP improves clinical and laboratory symptoms, reduces viral loads, thus allowing discharge of patients. The results of these studies are summarized in Table 1. CP is a passive immune therapy approach historically used in anti-inflammatory therapy of patients infected with various microorganisms, including bacteria, fungi, and viruses [20]. For viral infection therapy, CP is used for the treatment of Ebola, influenza, MERS and SARS-CoV coronaviruses [21]. In CP therapy, the collected plasma from survived patients is enriched with immunization factors, including immunoglobulins and high concentrations of antibodies to enhance its therapeutic effects through mechanisms, including neutralization of virus infectivity, enhancing recovery of the immune system, and inducing immediate or durable immunity.

\section{Discussion}

Low dose radiation can modulate inflammatory responses

Low dose radiation therapy (LDRT) was historically used to treat pneumonia during the first half of the $20^{\text {th }}$ century [22]. The anti-inflammatory effects of LDRT are well known. Given this consideration, Ghadimi-Moghadam et al. suggested that LDRT (at radiation doses of $0.10,0.18$ or $0.25 \mathrm{~Gy}$ ) to the lungs may reduce inflammation in pneumonia caused by SARS-CoV-2 [23]. If the innate immune is effectively activated during infection of the respiratory system, SARS-CoV-2 would efficiently be eliminated. Therefore, it would not enter the blood. In this situation, the infected patient does not show clinical signs nor exhibits symptoms of COVID-19. However, when the immune responses fail, the virus will enter the blood and cause tissue damage in ACE2expressing tissues and cells. Overall, the viral replication, SARS-CoV-2-mediated apoptosis and pyroptosis, host inflammatory responses, and cytokine storm may contribute to the COVID-19 pathogenesis. Low dose radiation can trigger beneficial effects for alleviation of COVID-19 through anti-inflammatory responses and optimization of the activity of the immune system. Excess release of pro-inflammatory cytokines such as IFN- $\alpha$, IFN- $\gamma$, IL- $1 \beta$, IL6, IL-12, IL-18, IL-33, TNF- $\alpha$, TGF $\beta$, and chemokines CCL2, CCL3, CCL5, CXCL8, CXCL9, CXCL10 from the immune effector cells causes hyperinflammation, which eventually leads to ARDS [1].

It has long been known that LDR can induce anti-inflammatory responses. Historically, LDRT was used for the treatment of infectious diseases, such as pneumonia and pertussis. The concept of using LDRT for COVID-19 pneumonia (Figure 1) was first introduced by a group of Iranian and American scientists in March 2020 [23]. Later Canadian [24], Spanish [25], American [26], German [27] and more recently French [28] scientists also confirmed the potential efficacy of LDRT for COVID-19 pneumonia. These studies focused on anti-inflammatory and immunomodulatory effects of LDRT and suggested that doses ranging from $100 \mathrm{mGy}$ to $1000 \mathrm{mGy}$ could be beneficial for treatment of severe pneumonia in COVID-19 


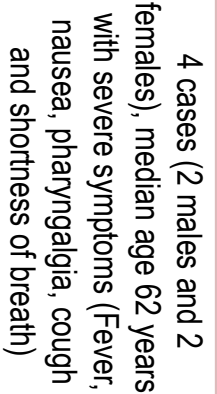

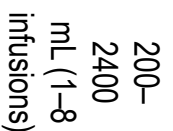

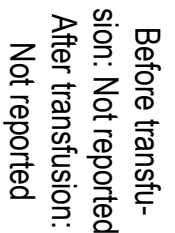

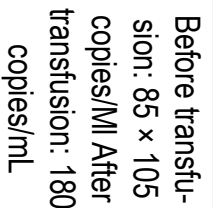

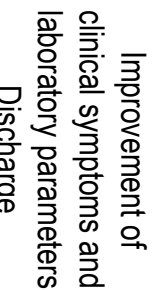

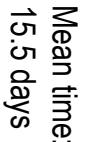

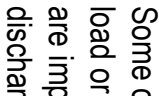

言 흥

变素

言 응

응ㅎㅇㅇ

要

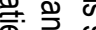

के

$\vec{\Phi}$

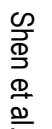

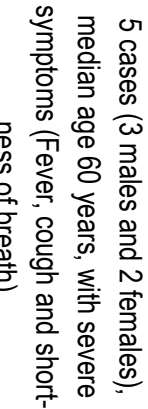

홍

эำ
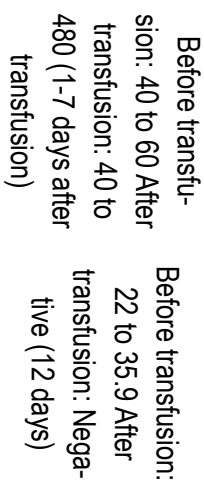

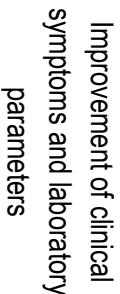

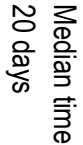

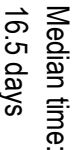

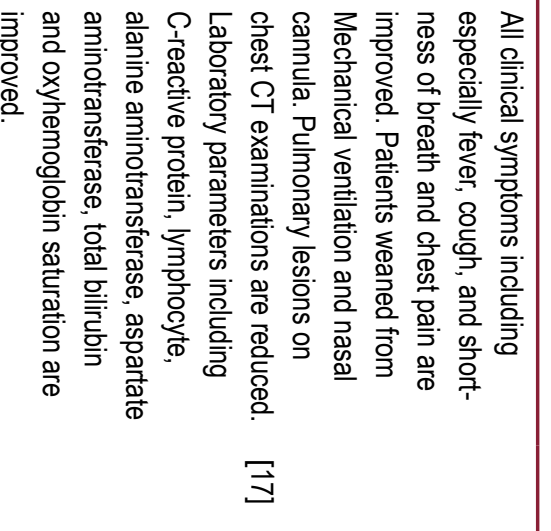

$\frac{\overrightarrow{2}}{\frac{5}{5}}$

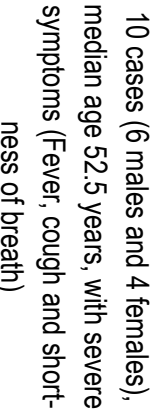

옳

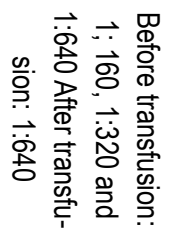

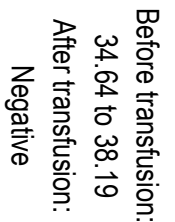

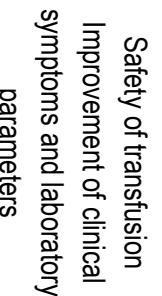

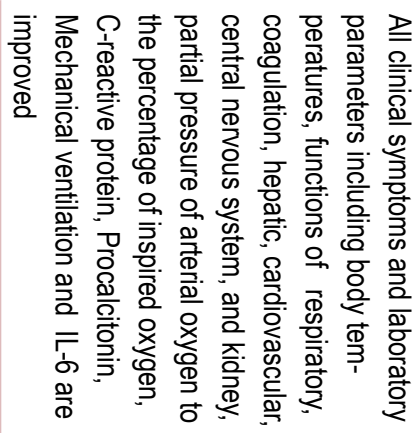

$\overrightarrow{\vec{\sigma}}$

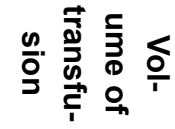

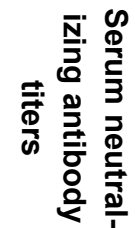

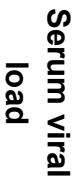

署

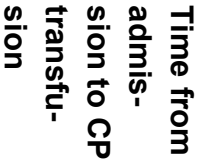

蛋

茴

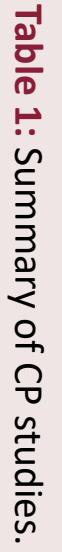

. 


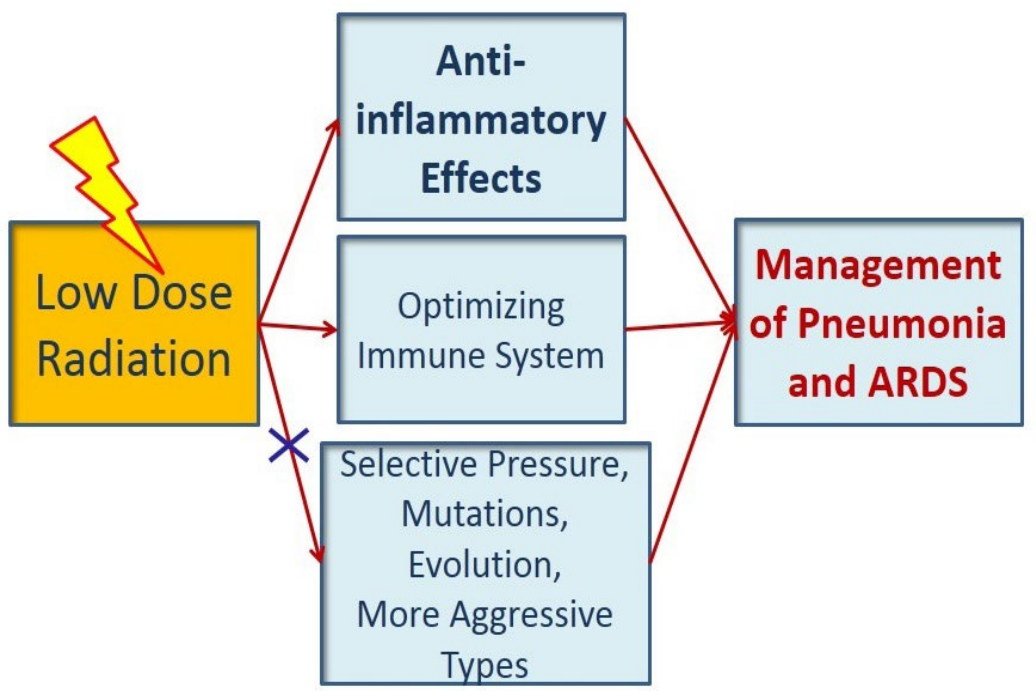

Figure 1: Possible mechanisms involved in the utilization of LDRT for the management of ARDS in COVID-19 patients.

patients.

Moreover, a wide array of global studies have been conducted to investigate the impact of LDR on the immune system, involving different levels of radiation exposure. Several studies indicated that LDR can enhance the immune system response. A review by Hekim et al., indicated that radiation-induced immunity could be obtained by altered expression of several genes involved in the immune-system signaling pathways [29]. The authors showed that LDR increases interferon- $\gamma$ production, activates natural killer cells, stimulates antigen processing and antigen presentation to T- cells, and activates natural killer T-cells (NKT), $\gamma \delta \mathrm{T}$ cells, and $\alpha \beta$ CD8 + T-cells. In addition, LDR has been shown to demonstrate significant effects on the secretion of pro-inflammatory cytokines, such as IL-8 and G-CSF.

In less than a month, the report by Ghadimi-Moghadam et al. [23] received attention not only for introducing LDRT as a treatment technique for pneumonia in COVID-19 patients but also for noting the key disadvantages of other treatment methods such as antiviral drugs. Rodel et al. [27] recently stated "SARS-COV-2 is an RNA virus with an ex- pected moderate to high mutation rate similar to other SARS RNA viruses and usually higher than the corresponding rate of the human host cells [30]. In addition, as discussed in a recent manuscript [23], any antiviral drug treatment against SARS-CoV-2 would probably result in a more intense selective pressure on the virus". Moreover Dilucca et al. highlighted the importance of LDRT and address the potential problems associated with the widespread use of different antiviral drugs "In addition, an interesting potential idea for the treatment of pneumonia-related to SARS-CoV-2 and other similar viruses is a low dose of ionizing radiation (LDIR). SARS-COV-2 is an RNA virus with an expected mutation rate similar to other RNA viruses, as discussed above. This mutation rate is usually much higher than the corresponding one of any human host. Therefore, as discussed in a recent paper [23], any antiviral drug against SARS-CoV-2 would exert an intense selective pressure on the virus. This may result in highly adaptive and treatmentresistant virus types with enhanced pathogenicity". It is worth noting that currently, there is no effective antiviral therapy available for SARS-CoV-2 infection, which mostly leads to 
life threatening inflammatory responses and acute lung injuries. [31].

In this work, we propose combination therapy involving both CP and LDR. We hypothesize that the immunomodulatory and antiinflammatory effects of radiation and plasma will synergistically impact COVID-19 patients. This combination therapy could activate both radiation and plasma pathways, and possibly new immune-system related pathways for better management of COVID-19. To conduct this therapeutic strategy, the following scenarios could be studied:

1. Low dose irradiation of chest area of CP donors prior to donation (not recommended due to ethical issues).

2. Low dose irradiation of COVID-19 patients' lungs few hours before CP transfusion.

3. Low dose irradiation of COVID-19 patients a few hours after CP transfusion.

In the first strategy, after irradiation of the chest area of CP donors, the provided plasma is used for treatment. However, due to ethical considerations, healthy donors should not be exposed even to low levels of radiation. The second option seems practical, since COVID-19 patients are irradiated prior to or following CP transfusion. Additional options that could potentially maximize the synergistic effects or provide guidance for additional treatment are CP transfusion, followed by LDRT, and then a subsequent $\mathrm{CP}$ transfusion as well as LDRT, followed by $\mathrm{CP}$ transfusion, and then followed by a subsequent LDRT administration.

\section{Conclusion}

$\mathrm{CP}$ has emerged as a potential antiviral and immunomodulatory therapy option for COVID-19. Recently, the immunomodulatory and inflamma-modulatory effects of LDR in COVID-19 have also been proposed. CP and LDR have significant potential to therapeutically impact COVID-19 through a positive synergistic interaction. We believe that this hybrid treatment framework has the potential to help healthcare providers in mitigating the adverse health effects of COVID-19.

\section{Conflict of Interest}

None

\section{References}

1. Astuti I. Severe Acute Respiratory Syndrome Coronavirus 2 (SARS-CoV-2): An overview of viral structure and host response. Diabetes \& Metabolic Syndrome: Clinical Research \& Reviews. 2020;14(4):407-12. doi: 10.1016/j. dsx.2020.04.020. PubMed PMID: 32335367. PubMed PMCID: PMC7165108.

2. Abdollahi $H$. Less is more: Intelligent intensive care for SARS-CoV-2 based on the imaging data. J Med Imaging Radiat Sci. 2020;51(2):198-9. doi: 10.1016/j.jmir.2020.04.002. PubMed PMID: 32340895. PubMed PMCID: PMC7165288.

3. Rothan HA, Byrareddy SN. The epidemiology and pathogenesis of coronavirus disease (COVID-19) outbreak. J Autoimmun. 2020;109:102433. doi: 10.1016/j.jaut.2020.102433. PubMed PMID: 32113704. PubMed PMCID: PMC7127067.

4. Sarzi-Puttini P, Giorgi V, Sirotti S, Marotto D, Ardizzone S, Rizzardini G, Antinori S, Galli M. COVID-19, cytokines and immunosuppression: what can we learn from severe acute respiratory syndrome? Clin Exp Rheumatol. 2020;38(2):337-42. PubMed PMID: 32202240.

5. Harapan $H$, Itoh N, Yufika A, Winardi W, Keam $\mathrm{S}$, Te $\mathrm{H}$, Megawati $\mathrm{D}$, Hayati $\mathrm{Z}$, Wagner $\mathrm{AL}$, Mudatsir M. Coronavirus disease 2019 (COVID-19): A literature review. J Infect Public Health. 2020;13(5): 667-73. doi: 10.1016/j. jiph.2020.03.019. PubMed PMID: 32340833. PubMed PMCID: PMC7142680.

6. Zhou F, Yu T, Du R, Fan G, Liu Y, Liu Z, Xiang J, Wang $Y$, Song B, Gu X, Guan L. Clinical course and risk factors for mortality of adult inpatients with COVID-19 in Wuhan, China: a retrospective cohort study. Lancet. 2020;395(10229):105462. doi: 10.1016/S0140-6736(20)30566-3. PubMed PMID: 32171076. PubMed PMCID: PMC7270627.

7. Gosain R, Abdou Y, Singh A, Rana N, Puzanov I, Ernstoff MS. COVID-19 and cancer: a comprehensive review. Curr Oncol Rep. 2020;22(5):53. doi: 10.1007/s11912-020-00934-7. PubMed PMID: 32385672. PubMed PMCID: PMC7206576.

8. Letko M, Marzi A, Munster V. Functional assess- 
ment of cell entry and receptor usage for SARSCoV-2 and other lineage B betacoronaviruses. Nat Microbiol. 2020;5(4):562-9. doi: 10.1038/ s41564-020-0688-y. PubMed PMID: 32094589. PubMed PMCID: PMC7095430.

9. Guan WJ, Ni ZY, Hu Y, Liang WH, Ou CQ, He JX, Liu L, Shan H, Lei CL, Hui DS, Du B, et al. Clinical characteristics of coronavirus disease 2019 in China. N Engl J Med. 2020;382(18):1708-20. doi: 10.1056/NEJMoa2002032. PubMed PMID: 32109013. PubMed PMCID: PMC7092819.

10. Lin L, Lu L, Cao W, Li T. Hypothesis for potential pathogenesis of SARS-CoV-2 infection-a review of immune changes in patients with viral pneumonia. Emerg Microbes Infect. 2020;9(1):727732. doi: 10.1080/22221751.2020.1746199. PubMed PMID: 32196410. PubMed PMCID: PMC7170333.

11. Xu H, Zhong L, Deng J, Peng J, Dan H, Zeng X, $\mathrm{Li}$ T, Chen $Q$. High expression of ACE2 receptor of 2019-nCoV on the epithelial cells of oral mucosa. Int J Oral Sci. 2020;12(1):8. doi: 10.1038/ s41368-020-0074-x. PubMed PMID: 32094336. PubMed PMCID: PMC7039956.

12. Vellingiri $B$, Jayaramayya $K$, Iyer $M$, Narayanasamy A, Govindasamy V, Giridharan B, Ganesan S, Venugopal A, Venkatesan D, Ganesan H, Rajagopalan K. COVID-19: A promising cure for the global panic. Sci Total Environ. 2020;725:138277. doi: 10.1016/j.scitotenv.2020.138277. PubMed PMID: 32278175. PubMed PMCID: PMC7128376.

13. Baig AM, Khaleeq A, Ali U, Syeda H. Evidence of the COVID-19 virus targeting the CNS: tissue distribution, host-virus interaction, and proposed neurotropic mechanisms. ACS Chem Neurosci. 2020;11(7):995-998. doi: 10.1021/ acschemneuro.0c00122. PubMed PMID: 32167747. PubMed PMCID: PMC7094171.

14. Zhang D, Guo R, Lei L, Liu H, Wang Y, Wang $Y$, et al. COVID-19 infection induces readily detectable morphological and inflammation-related phenotypic changes in peripheral blood monocytes, the severity of which correlate with patient outcome. medRxiv. 2020. doi: 10.1101/2020.03.24.20042655.

15. Mair-Jenkins J, Saavedra-Campos M, Baillie JK, Cleary P, Khaw FM, Lim WS, Makki S, Rooney KD, Convalescent Plasma Study Group, Nguyen-Van-Tam JS, Beck CR. The effectiveness of convalescent plasma and hyperimmune immunoglobulin for the treatment of severe acute respiratory infections of viral etiology: a systematic review and exploratory meta-analysis. $J / n$ fect Dis. 2015;211(1):80-90. doi: 10.1093/infdis/ jiu396. PubMed PMID: 25030060. PubMed PMCID: PMC4264590.

16. Shen C, Wang Z, Zhao F, Yang Y, Li J, Yuan J, Wang F, Li D, Yang M, Xing L, Wei J. Treatment of 5 critically ill patients with COVID-19 with convalescent plasma. Jama. 2020;323(16):1582-9. doi: 10.1001/jama.2020.4783. PubMed PMID: 32219428. PubMed PMCID: PMC7101507.

17. Duan K, Liu B, Li C, Zhang H, Yu T, Qu J, Zhou M, Chen L, Meng S, Hu Y, Peng C. Effectiveness of convalescent plasma therapy in severe COVID-19 patients. Proceedings of the National Academy of Sciences. 2020;117(17):9490-6. doi: 10.1073/pnas.2004168117.

18. Zhang B, Liu S, Tan T, Huang W, Dong Y, Chen L, Chen $Q$, Zhang L, Zhong Q, Zhang X, Zou $Y$. Treatment with convalescent plasma for critically ill patients with SARS-CoV-2 infection. Chest. 2020. doi: 10.1016/j.chest.2020.03.039. PubMed PMID: 32243945. PubMed PMCID: PMC7195335.

19. US Food and Drug Administration. Donate COVID-19 Plasma. US FDA; 2020.

20. Brown BL, McCullough J. Treatment for emerging viruses: Convalescent plasma and COVID-19. Transfus Apher Sci. 2020;59(3):102790. doi: 10.1016/j.transci.2020.102790. PubMed PMID: 32345485. PubMed PMCID: PMC7194745.

21. Sullivan HC, Roback JD. Convalescent plasma: therapeutic hope or hopeless strategy in the SARS-CoV-2 pandemic. Transfus Med Rev. 2020. doi: 10.1016/j.tmrv.2020.04.001. PubMed PMID: 32359788. PubMed PMCID: PMC7179481.

22. Calabrese EJ, Dhawan G. How radiotherapy was historically used to treat pneumonia: could it be useful today? Yale J Biol Med. 2013;86(4):555. PubMed PMID: 24348219. PubMed PMCID: PMC3848110.

23. Ghadimi-Moghadam $A$, Haghani $M$, Bevelacqua JJ, Jafarzadeh A, Kaveh-Ahangar A, Mortazavi SMJ, Ghadimi-Moghadam A, Mortazavi SAR. COVID-19 tragic pandemic: concerns over unintentional "directed accelerated evolution" of novel Coronavirus (SARS-CoV-2) and introducing a modified treatment method for ARDS. J Biomed Phys Eng. 2020; 10(2):241-6. doi: 10.31661/ jbpe.v0i0.2003-1085. PubMed PMID: 32337192. PubMed PMCID: PMC7166223. 
24. Kirkby C, Mackenzie M. Is low dose radiation therapy a potential treatment for COVID-19 pneumonia? Radiother Oncol. 2020. doi: 10.1016/j. radonc.2020.04.004. PMID: 32342871. PMCID: PMC7194710.

25. Lara PC, Burgos J, Macias D. Low dose lung radiotherapy for COVID-19 pneumonia. The rationale for a cost-effective anti-inflammatory treatment. Clin Transl Radiat Oncol. 2020;23:27-9. doi: 10.1016/j.ctro.2020.04.006. PubMed PMID: 32373721. PubMed PMCID: PMC7193092.

26. Dhawan G, Kapoor R, Dhawan R, Singh R, Monga B, Giordano J, Calabrese EJ. Low dose radiation therapy as a potential life saving treatment for COVID-19-induced acute respiratory distress syndrome (ARDS). Radiother Oncol. 2020 ;147:212-6. doi: 10.1016/j.radonc.2020.05.002. PubMed PMID: 32437820. PubMed PMCID: PMC7206445.

27. Rödel F, Arenas M, Ott OJ, Fournier C, Georgakilas AG, Tapio S, Trott KR, Gaipl US. Low-dose radiation therapy for COVID-19 pneumopathy: what is the evidence? Strahlenther Onkol. 2020;14. doi: 10.1007/s00066-020-01635-7. doi: 10.1007/s00066-020-01635-7. PubMed PMID:
32388805. PubMed PMCID: PMC7211051.

28. Cosset JM, Deutsch É, Bazire L, Mazeron JJ, Chargari C. Irradiation pulmonaire à faible dose pour l'orage de cytokines du COVID-19: pourquoi pas? Cancer Radiother. 2020;24(3):179-81. doi: 10.1016/j.canrad.2020.04.003. PubMed PMID: 32389579. PubMed PMCID: PMC7252150.

29. Hekim N, Cetin Z, Nikitaki Z, Cort A, Saygili El. Radiation triggering immune response and inflammation. Cancer Lett. 2015;368(2):156-63. doi: 10.1016/j.canlet.2015.04.016. PubMed PMID: 25911239.

30.Zhang QF, Li YW, Liu ZH, Chen QL. Exposure to mercuric chloride induces developmental damage, oxidative stress and immunotoxicity in zebrafish embryos-larvae. Aquat Toxicol. 2016;181:76-85. doi: 10.1016/j.aquatox.2016.10.029. PubMed PMID: 27821350.

31. Fu Y, Cheng Y, Wu Y. Understanding SARSCoV-2-mediated inflammatory responses: from mechanisms to potential therapeutic tools. Virologica Sinica. 2020:1-6. doi: 10.1007/s12250020-00207-4. PubMed PMID: 32125642. PubMed PMCID: PMC7090474. 